\title{
USO DA TERMOGRAFIA INFRAVERMELHA NA IDENTIFICAÇÃO DE DOR EM TRABALHADORES ENCAMINHADOS PARA REABILITAÇÃO
}

\author{
Márcio Alves Marçal (1); \\ Ana Paula Vieira Elias (2); \\ Fernanda Fabiane Dumont e Silva (3)
}

(1) Universidade Federal dos Vales do Jequitinhonha e Mucuri - UFVJM, Ph.D.

E-mail:marcio@nersat.com.br

(2) Universidade Federal dos Vales do Jequitinhonha e Mucuri - UFVJM - Graduação

E-mail: ana_paulinha_vieira@hotmail.com

(3) Núcleo de Ergonomia, Saúde e Segurança do Trabalho - NERSAT, Graduação.

E-mail: fernandadumont@nersat.com.br

\begin{abstract}
RESUMO
A reabilitação profissional é de extrema importância nos Distúrbios Osteomusculares Relacionados ao Trabalho (DORT), tendo em vista o alto índice de afastamento para tratamento, e possuindo como fator limitante a dor. E como esta é subjetiva e influenciada por fatores psicológicos, torna-se um desafio conseguir mensurá-la. A termografia é um procedimento diagnóstico que mensura a energia infravermelha emitida pelo corpo através de imagens de alta resolução constituindo a base para um diagnóstico funcional da dor. A imagem termográfica é uma boa ferramenta para avaliação da queixa de dor de maneira objetiva nos funcionários em tratamento na fisioterapia ortopédica e traumatológica.
\end{abstract}

Palavras-chave: dor, termografia infravermelha, DORT.

\begin{abstract}
Occupational rehabilitation is extremely important in Work-Related Musculoskeletal Disorders (MSDs), given the high absenteeism rate for treatment, and having pain as limiting factor. Pain is subjective and influenced by psychological factors, it becomes a challenge to measure it. Thermography is a diagnostic procedure that measures the infrared energy emitted by the body through high-resolution images forming the basis for a functional diagnosis of pain. The thermographic image is a good tool to evaluate objectively pain complaint in employees being treated in orthopedic and trauma therapy.
\end{abstract}

Key-words: pain, infrared thermography, MSDs. 


\section{INTRODUÇÃO}

A reabilitação profissional é de extrema importância quando se fala sobre saúde do trabalhador e principalmente nos casos dos Distúrbios Osteomusculares Relacionados ao Trabalho (DORT), tendo em vista o alto índice de afastamento para tratamento (Lima et al., 2010). A reabilitação do funcionário deve ser um conjunto integrado de ações com a finalidade de resgatar as capacidades física, psicológica e social quando estes são acometidos por agravos de saúde, que resultam na incapacidade para o trabalho (Takahashi et al., 2010). Na clínica escola da Universidade Federal dos Vales do Jequitinhonha e do Mucuri (UFVJM) funcionários procuram atendimento necessitando de tratamento para reabilitação de patologias ortopédicas de origem ocupacional. O principal fator limitante é a queixa de dor que dificulta e prolonga a reabilitação. O primeiro objetivo do tratamento é a redução da dor permitindo a evolução desse funcionário, porém o grande desafio é ter instrumentações que permitam a obtenção de indicadores mais objetivos evitando a subjetividade das queixas dos pacientes.

A dor é uma experiência comumente associada à lesão, e é de grande abrangência na prática clínica, sendo por muitas vezes o motivo que leva o indivíduo a procurar auxílio no sistema de saúde. A sensação dolorosa pode ser definida como uma experiência sensorial e emocional desagradável associada a um dano real ou potencial de tecidos, ou descrita em termos de tal dano de acordo com a Associação Internacional para o Estudo da Dor, (IASP International Association for the Study of Pain). A avaliação quantitativa da dor na aplicação clínica é algo desafiador, devido ao seu componente emocional, que torna a sensação dolorosa algo subjetivo sendo influenciada por fatores psicológicos que geram uma resposta única para cada indivíduo (McCaffery e Beebe, 1989). A mensuração da dor tem sido considerada um grande desafio para aqueles que desejam controlá-la adequadamente, pois a dor é entendida como uma experiência de percepção complexa, individual e subjetiva que pode ser quantificada apenas indiretamente como descrito por Hortense et al. (2008).

Os métodos mais utilizados na prática clínica são as descrições verbais, como ressalta Scopel et al. (2007), como exemplos desses métodos tem-se as escalas de dor, entrevistas e diários de dor que permitem a compreensão da subjetividade do paciente e de diversas outras variáveis não acessadas pelos dois outros métodos. Há também o Imageamento Infravermelho (IR), que é um procedimento diagnóstico que mensura a energia infravermelha emitida pelo corpo. Estas medidas se expressam na forma de imagens de alta resolução que constituem a base para serem utilizadas no diagnóstico de condições dolorosas. A termografia infravermelha computadorizada pode capturar o calor produzido pelo corpo humano invisível a olho nu, e tem capacidade de detectar mudanças térmicas de $0,05^{\circ} \mathrm{C}$ a $0,1^{\circ} \mathrm{C}$, captando as imagens infravermelhas e organizando-as em um mapa térmico (BRIOSCHI et al., 2005).

A presença de dor pode modificar o fluxo sanguíneo e esse pode ser detectado pela monitoração infravermelha, o que fornece um excelente método para correlacionar com a percepção da dor e documentar em imagens em tempo real (LOVE,1980). A distribuição da temperatura da pele de um corpo humano saudável apresenta uma simetria bilateral (HOUDAS, 2013). A distribuição de temperatura que mostra padrões assimétricos geralmente é um forte indicador de anormalidade (GOODMAN, 1986). Uma vez que a dissipação de calor através da pele ocorre, na maioria das vezes, sob a forma de radiação 
infravermelha, a termografia infravermelha é o método de escolha para o estudo da fisiologia e de termorregulação da disfunção térmica associada com dor (BRIOSCHI et al., 2005).

\section{OBJETIVO}

Avaliar se a imagem termográfica pode ser boa indicadora de dor comparada à avaliação subjetiva de queixa de dor em funcionários encaminhados para reabilitação ortopédica e traumatológica.

\section{METODOLOGIA}

A coleta ocorreu na clínica escola de fisioterapia, em uma sala aclimatizada e para monitorar as condições de temperatura e ventilação da mesma foi usado o Termoanemômetro Digital da Instruterm.

Foi demarcado um quadrado no chão do laboratório tendo, ao fundo, uma parede branca para que todos para padronização da posição dos funcionários estudados. A partir deste ponto, tinha uma linha reta no piso feita com adesivo de 2 metros de comprimento. Esta marcação permitiu uma padronização da distância do trabalhador e do tripé com a câmera durante os exames. A figura 1 ilustra esse posicionamento.

Figura 1: Posicionamento do trabalhador para registro das imagens termográficas.

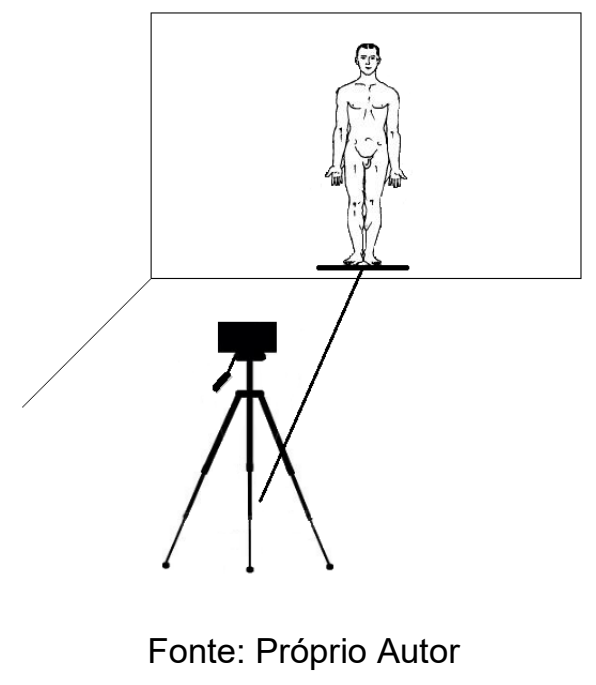

As avaliações foram realizadas por pesquisadores devidamente treinados. Todos os funcionários foram selecionados de maneira voluntária e foram submetidos aos seguintes procedimentos: os indivíduos permaneceram por 15 minutos na sala para que ocorresse o equilíbrio térmico, antes de se iniciar o processo de aquisição das imagens, com a região corporal a ser fotografada desnuda. Neste período responderam ao questionário sobre a localização e a intensidade da dor. O questionário era formado pelo desenho do corpo humano, de frente e de costas, e pela Escala Visual Analógica de Dor (EVA). Foi solicitado ao paciente colorir no desenho o local da queixa de dor e, em seguida, que marcasse na $E V A$, com um ' $X$ ', o local que melhor definisse a intensidade da dor que sentiam naquele momento em uma linha de $10 \mathrm{~cm}$ com numeração variando de 0 (zero) a 10 (dez). Após, 
todo esse preenchimento e passado o período de aclimatização, os trabalhadores foram colocados no local predeterminado para realização das fotos.

\section{RESULTADOS}

Para a coleta das imagens termográficas a sala permaneceu com temperatura local entre $19^{\circ}$ a $20^{\circ} \mathrm{C}$, umidade relativa do ar variando de $55 \%$ a $60 \%$ e ventilação do ar zero não havendo registro no Termoanemômetro.

O estudo contou com a participação de 20 indivíduos, mas dois deles foram excluídos do estudo. Um devido ao diagnóstico de osteomalácia tumoral, relatado no dia da coleta, e como esta patologia modifica de maneira geral a temperatura corporal global, logo não permitiria uma coleta fidedigna. $O$ outro foi excluído pela baixa qualidade das imagens coletadas visto que não permitiram sua análise. Nos 18 voluntários restantes, é possível perceber uma heterogeneidade da amostra, composta por 13 do sexo feminino e 5 do sexo masculino, idade média de $42 \pm 14,03$.

$\mathrm{Na}$ escala visual de dor, que varia de 1 a 10 onde 1 significa ausência de dor e 10 dor intensa, os pacientes registraram um valor médio de $5,20 \pm 2,81$. Os indivíduos apresentaram queixas variadas, independente do seu diagnóstico clínico, sendo que o mesmo indivíduo apresentou mais de um local com queixa dolorosa, totalizando 33 pontos de queixas de dor. As regiões do corpo onde foram relatadas as algias e o número de indivíduos que as relataram podem ser vistas no gráfico 1 . O gráfico ilustra que o local de maior queixa foi à coluna lombar, seguido dos joelhos e tornozelos.

Gráfico 1: Local da queixa dolorosa em relação ao número de indivíduos.

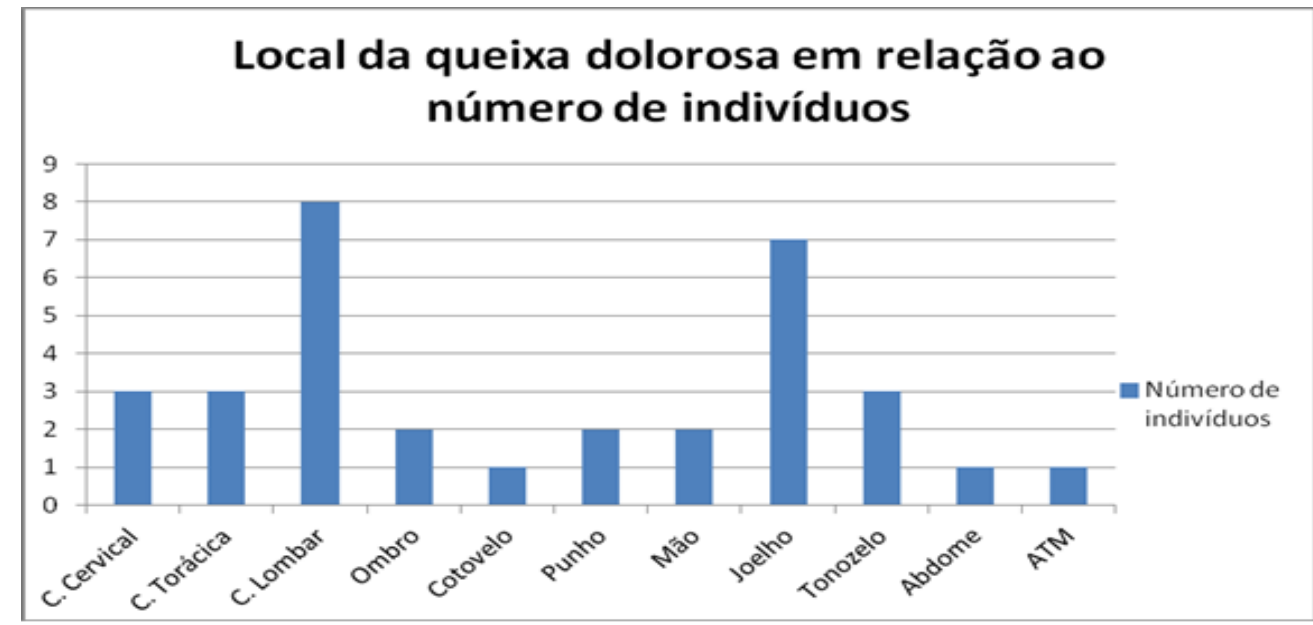

Fonte: Próprio Autor

Do total de 33 imagens termográficas analisadas, 28 apresentaram alterações funcionais nas regiões demarcadas nos desenhos pelos funcionários. Correspondendo a um acerto de $84,8 \%$ entre imagens e áreas marcadas no desenho. 
Abaixo estão apresentados exemplos ilustrando as imagens termográficas coletadas com identificação de alterações funcionais nos pontos de queixa de dor marcados, no questionário, pelos trabalhadores. Ressaltando que foi usado como padrão para quantificação da intensidade das alterações funcionais dos voluntários o valor obtido pelo Delta $T(\Delta T)$, que é o parâmetro usado na comparação de uma determinada região de interesse (ROI), em relação ao seu homólogo contralateral. A Tabela 1 mostra a quantificação do $\Delta \mathrm{T}$ e os indicadores de acordo com cada faixa desses valores.

Tabela 1: Equiparação entre valor encontrado pelo $\Delta \mathrm{T}$ e seus indicadores.

\begin{tabular}{|c|c|}
\hline Delta $T(\Delta T)$ & Indicadores \\
\hline$<0,24^{\circ} \mathrm{C}$ & NORMAL \\
\hline$>0,3^{\circ} \mathrm{C}$ até $0,6^{\circ} \mathrm{C}$ & SUGESTIVO DE ANORMALIDADE \\
\hline$>0,6^{\circ} \mathrm{C}$ ATÉ $1,0^{\circ} \mathrm{C}$ & FORTEMENTE SUGESTIVO \\
\hline$>1,0^{\circ} \mathrm{C}$ & ANORMALIDADE SIGNIFICATIVA \\
\hline
\end{tabular}

Fonte: Manual de Termografia Médica.

O primeiro exemplo mostra uma funcionária com doença ocupacional relacionada ao trabalho (DORT) que possui queixas de dor na coluna cervical, tendo maior prevalência no lado esquerdo e na coluna lombar, com maior predomínio no lado direito. Na vista posterior das costas da paciente percebe-se que a região da coluna cervical possui maior temperatura no lado esquerdo $\left(\Delta \mathrm{T}=0,5^{\circ} \mathrm{C}\right)$ e na região lombar, em vista mais aproximada, a temperatura é maior do lado direito em relação ao esquerdo $\left(\Delta \mathrm{T}=0,9^{\circ} \mathrm{C}\right)$. A figura 2 ilustra as imagens termográficas captadas e o desenho feito no questionário pela voluntária. Valor da intensidade de queixa dolorosa no EVA: 6 (coluna cervical) e 8 (coluna lombar).

Figura 2: Imagens termográficas e desenho dos pontos de localização de dor

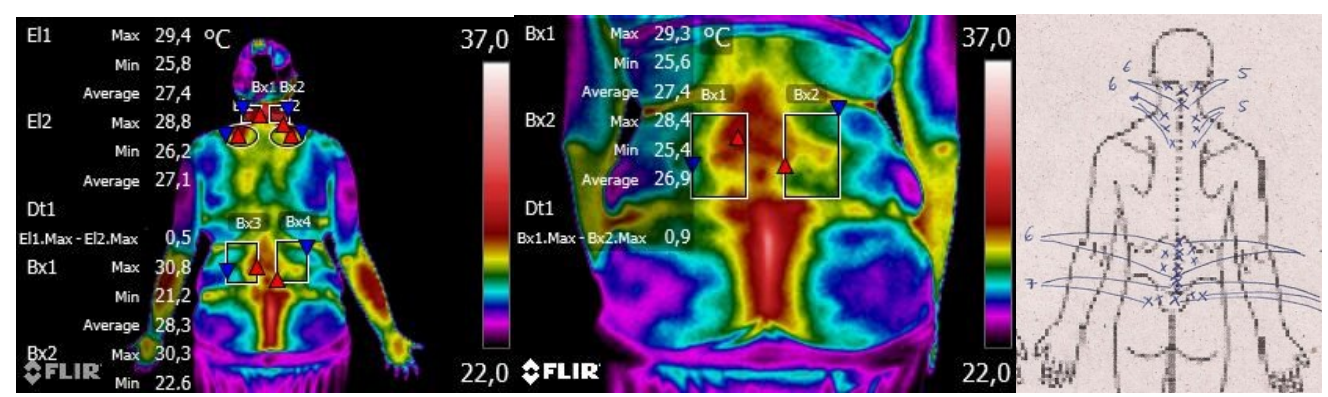

O segundo exemplo mostra outra funcionária, também com DORT, que possui queixa de dor na região do maléolo lateral do tornozelo direito. Nas visões laterais dos tornozelos da paciente fica visível a diferença de temperaturas locais (diferença de $1,5^{\circ} \mathrm{C}$ de temperatura entre o lado direito e esquerdo). A figura 3 ilustra as imagens termográficas captadas e o desenho feito no questionário pela voluntária. Valor da intensidade de queixa dolorosa no EVA: 8. 
Figura 3: Imagens termográficas e desenho dos pontos de localização de dor
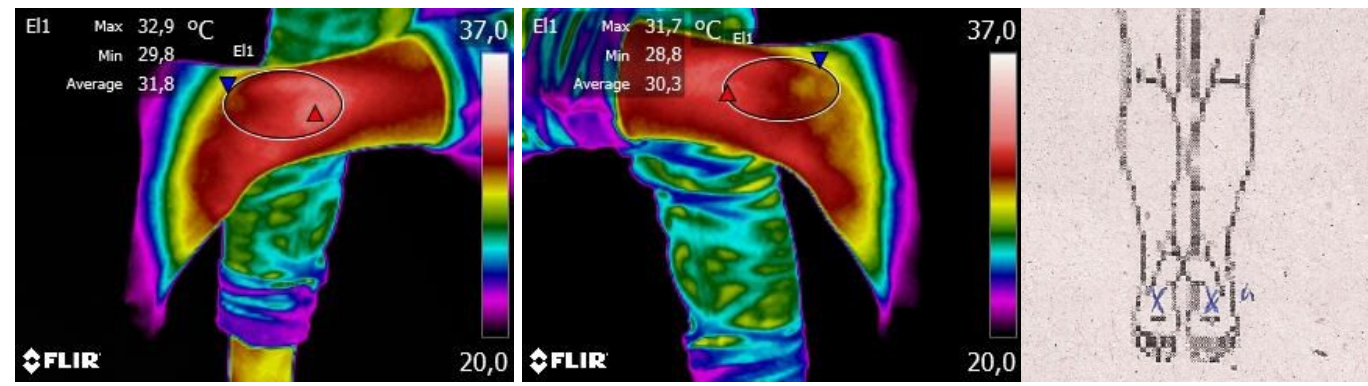

O exemplo 3 mostra um funcionário que teve acidente de moto, há seis meses, no trajeto para o trabalho, que resultou em fratura do osso escafoide e desde então possui dor na região do punho esquerdo. Na visão anterior dos punhos percebe-se que o processo no local de queixa de dor é crônico, pois o punho esquerdo possui temperatura inferior ao lado contralateral $\left(\Delta T=1,4^{\circ} \mathrm{C}\right)$. A figura 4 ilustra as imagens termográficas captadas e o desenho feito no questionário pelo trabalhador. Valor da intensidade de queixa dolorosa no EVA: 3.

Figura 4: Imagem termográfica e desenho dos pontos de localização de dor

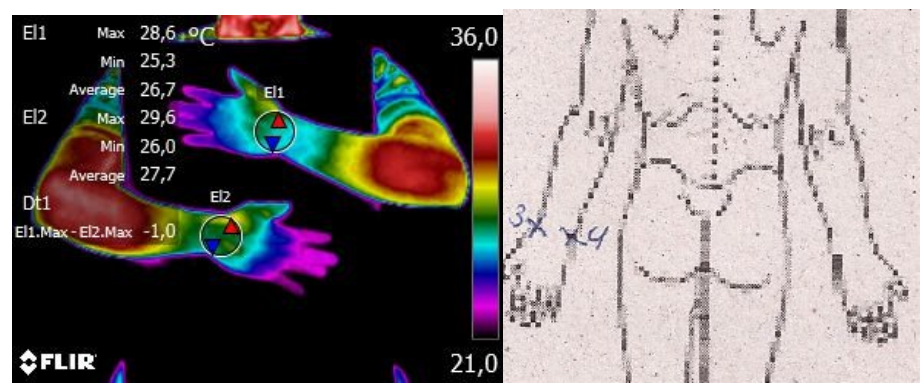

Já no exemplo 4, a trabalhadora possui DORT com queixa no membro inferior direito, sendo que o joelho e a perna possuem maior foco de dor. Na visão anterior, perna e pé direito mostram aumento metabólico sugerindo sobrecarga nesta região. É visível também que o joelho direito possui um aumento de temperatura local maior que o esquerdo $\left(\Delta T=0,5^{\circ} \mathrm{C}\right)$. A figura 5 ilustra as imagens termográficas captadas e o desenho feito no questionário por ela. Valor da intensidade de queixa dolorosa no EVA: 6 (joelho) e 5 (perna).

Figura 5: Imagem termográfica e desenho dos pontos de localização de dor

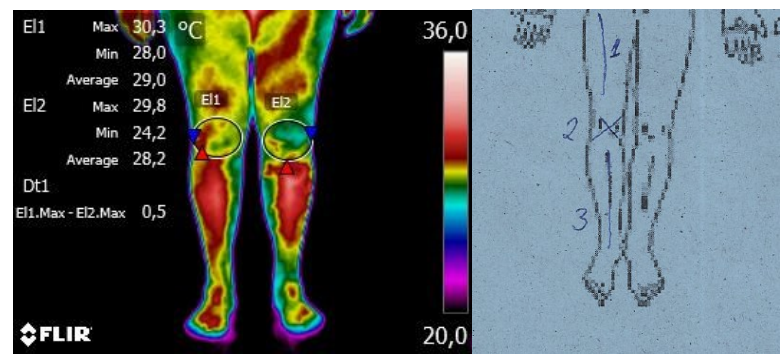

Esta funcionária do exemplo 5 relata queixa de dor na região da coluna lombar por DORT. $\mathrm{Na}$ vista posterior do tronco, na região lombar, percebe-se uma irradiação para o lado direito podendo indicar maior dor neste lado $(\Delta \mathrm{T}=0,7)$. A figura 6 ilustra as imagens termográficas captadas e o desenho feito no questionário pela voluntária. Valor da intensidade de queixa dolorosa no EVA: 8. 
Figura 6: Imagens termográficas e desenho dos pontos de localização de dor
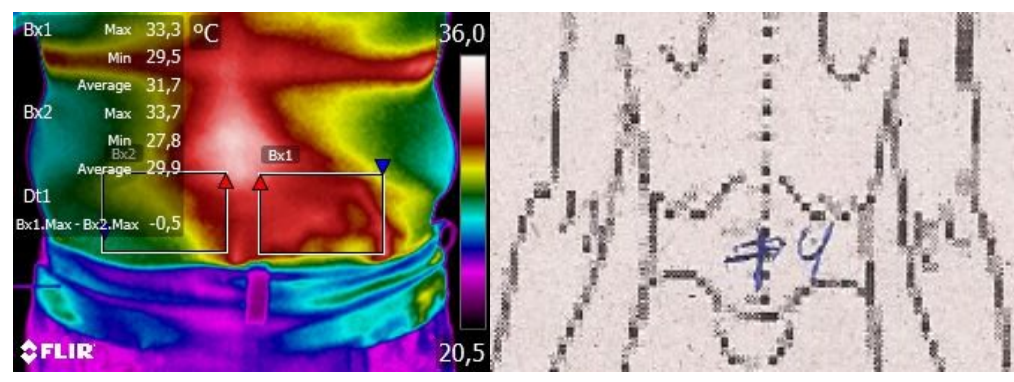

No exemplo 6 o funcionário apresenta dor no $3^{\circ}$ dedo e nos $2^{\circ} / 3^{\circ}$ metacarpos da mão esquerda, pós-fratura, por acidente de trabalho, já corregida de forma cirúrgica há 8 meses. $\mathrm{Na}$ imagem termográfica há uma clara marcação nos pontos de queixa de dor do mesmo demonstrando alteração funcional crônica em ambos os pontos, justificado pela apresentação de uma redução da temperatura nestes locais. A figura 7 ilustra as imagens termográficas captadas e o desenho feito no questionário pelo voluntário. Valor da intensidade de queixa dolorosa no EVA: 2 ( $2^{\circ} / 3^{\circ}$ metacarpos) e 5 ( $3^{\circ}$ dedo).

Figura 7: Imagens termográficas e desenho dos pontos de localização de dor
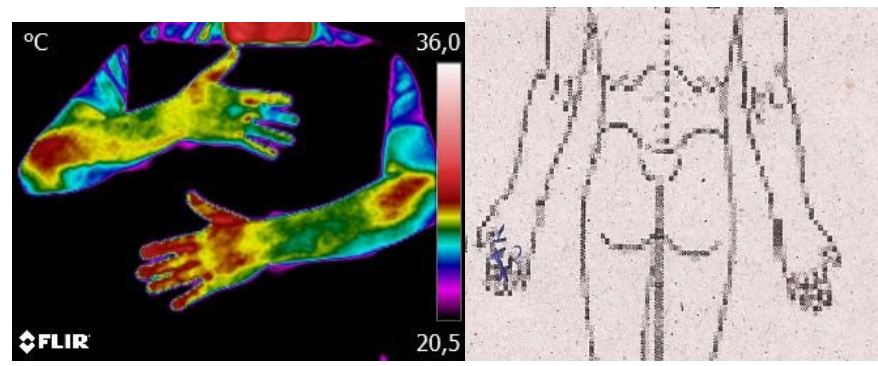

No exemplo 7 a funcionária apresenta DORT nas regiões do ombro e do cotovelo direitos com queixas de dor. $\mathrm{Na}$ visão anterior a imagem termográfica demonstra alteração na temperatura local do ombro direito indicativa de alteração funcional nesta região $(\Delta T=0,6$ ${ }^{\circ} \mathrm{C}$ ). Na mesma imagem é possível verificar também que há alteração funcional no cotovelo direito em relação ao esquerdo $\left(\Delta \mathrm{T}=1,7^{\circ} \mathrm{C}\right)$ indicando alteração aguda local que deve ser investigada. A figura 8 ilustra as imagens termográficas captadas e o desenho feito no questionário pela voluntária. Valor da intensidade de queixa dolorosa no EVA: 10, nas duas regiões.

Figura 8: Imagem termográfica e desenho dos pontos de localização de dor

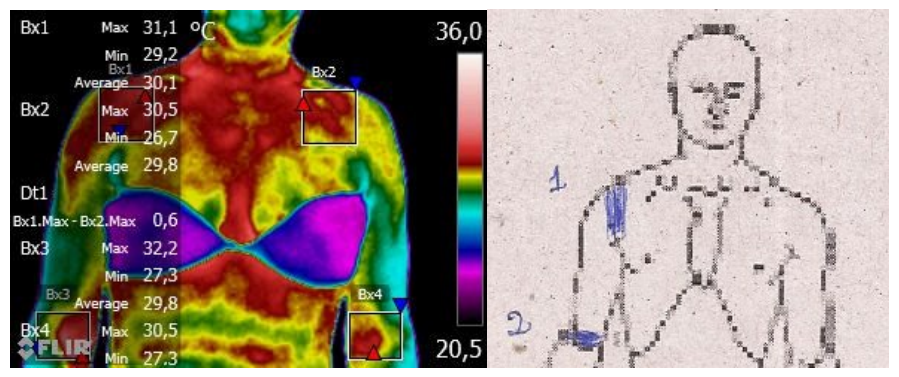

Os próximos exemplos (8 e 9) ilustram imagens termográficas que não mostram alterações funcionais nos pontos de queixa de dor marcados, pelos trabalhadores, no diagrama com a imagem do corpo humano. 
No exemplo 8 a funcionária relata queixa de dor na região lombar, mas apesar do aumento metabólico na região lombar não apresenta assimetria térmica. A hiperradiação na região é devido ao aumento da curvatura presente na região lombar mais as dobras da pele. A figura 9 ilustra as imagens termográficas captadas e o desenho feito no questionário pela mesma. Valor da intensidade de queixa dolorosa no EVA: 4.

Figura 9: Imagem termográfica e desenho dos pontos de localização de dor
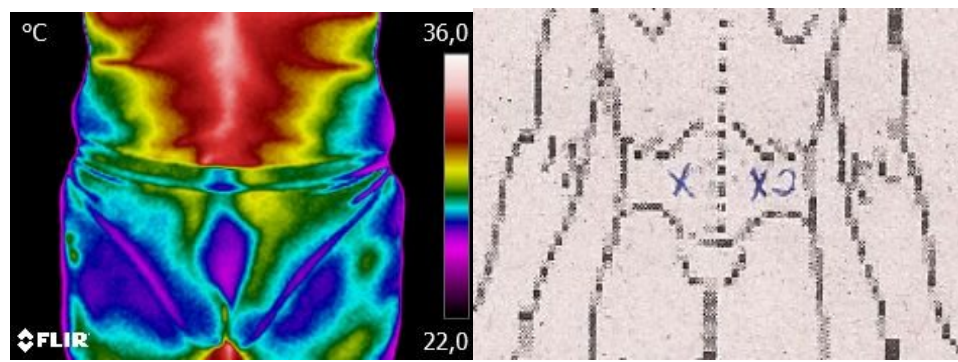

No exemplo 9 há relato de queixa de dor na região do punho esquerdo, mas a imagem termográfica captada mostra-se simétrica, portanto não apresenta sinal de alteração funcional. A figura 10 ilustra as imagens termográficas captadas e o desenho feito no questionário pela voluntária. Valor da intensidade de queixa dolorosa no EVA: 2.

Figura 10: Imagem termográfica e desenho dos pontos de localização de dor

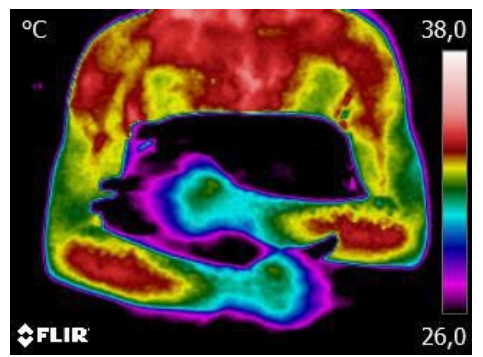

\section{DISCUSSÃO}

O presente estudo mostrou que a termografia infravermelha demonstrou uma diferença de temperatura local $(\Delta T)$ que foi condizente com o registro feito pelos funcionários no questionário em $84,84 \%$ das queixas de dor. Este resultado vai de encontro ao estudo de Neves et al. (2015) que relataram que a termografia é um bom instrumento de confirmação de pontos de queixa de dor sendo uma abordagem útil para o diagnóstico e acompanhamento de vários distúrbios físicos.

Geralmente, boa parte das lesões teciduais, inclusive das DORTs, relaciona-se com as variações no fluxo sanguíneo, podendo afetar a temperatura cutânea. As mesmas estão relacionadas com: alterações na densidade, composição, volume e temperatura do local afetado (DE MEIRA et al, 2014). A presença de dor também pode modificar o fluxo sanguíneo observado no local. A dor musculoesquelética é desencadeada pela ativação dos nociceptores periféricos. Eles estão presentes no centro da musculatura e em terminações nervosas livres dos aferentes primários das fibras do grupo III e do grupo IV, densamente condensados nos tendões, fáscias, cápsulas e aponeuroses (TEIXEIRA et al, 2001). No caso das DORTs a ativação desses nociceptores não é diferente, levando inicialmente à queixas de cansaço na região acometida, redução da capacidade de trabalho que, 
posteriormente, pode levar ao afastamento temporário ou definitivo do trabalhador. Portanto, a reabilitação eficaz e rápida é um importante aliado na recuperação do profissional.

Riley e Richte (1975) relatam que é possível demarcar diversos tipos de dores, assim como condições fisiológicas, pela mensuração da resistência elétrica cutânea. No estudo que realizaram as áreas de dores corresponderam às áreas de baixa resistência (hiperatividade simpática). Love (1980) observa ainda que o sistema nervoso neurovegetativo simpático (SNNVS) tem uma estreita relação com a dor, sendo possível documentar as alterações cutâneas causadas por fenômenos vasculares devido a reflexos neurovegetativos, na mesma área do corpo onde o paciente se queixa de dor. O leito vascular é controlado diretamente pelo SNNVS, logo, áreas de maior ou menor emissão podem ser correlacionadas com o funcionamento do mesmo. A presença de dor pode modificar o fluxo sanguíneo observado pela monitoração infravermelha. Isto fornece um excelente método para correlacionar com a percepção da dor e documentar em imagens em tempo real podendo ser obtido pelo imageamento IR, que é o melhor método de mensurar o fluxo sanguíneo superficial cutâneo (LOVE,1980).

A imagem IR pode diferenciar os dermátomos pelos reflexos neurovegetativos microvasculares da pele. Devido ao controle neurovegetativo, uma área cutânea do corpo é capaz de modificar sua resposta à dor e produzir certa energia térmica que é relacionada ao fluxo vascular. Isso pode resultar em maior (quente) ou menor (frio) radiação infravermelha no local, e estas áreas de mudança são denominadas termátomos, que são áreas assimétricas em relação ao lado contralateral correspondente do corpo e, em muitos casos, irão concordar com a localização da alteração neuronal e com o local da queixa de dor relatada (BRIOSCHI et al,2005). Como mencionado, pessoas com queixas de dor apresentam imagens IR anormais devido a alterações vasomotoras. Essas disfunções não podem ser demonstradas por estudos radiológicos convencionais, até que mudanças estruturais ocorram. As alterações IR estão mais relacionadas à função fisiológica do que com a morfologia em si, o que mostra a capacidade do imageamento em rastrear a presença de dor antes mesmo que a lesão se encontre instalada.

Neste trabalho foram encontradas 11 regiões de dor, sendo elas: região cervical, torácica, lombar, articulação temporomandibular, ombro, cotovelo, punho, mão, abdome, joelho e tornozelo; tendo 9 relacionadas à DORTs, uma a acidente de trabalho e uma a acidente de trajeto. A região lombar $(24,24 \%)$ e a região dos joelhos $(21,21 \%)$ foram as mais incidentes. A dor lombar possui a maior ocorrência dentre os distúrbios músculoesqueléticos, sendo relatada, em algum momento, por cerca de $80 \%$ da população em geral. (DEYO et al., 2006). No Brasil, 63\% da população adulta já relatou, em algum momento da vida, a ocorrência de dor lombar (FERREIRA et al., 2011), com predominância no sexo feminino (Matos et al., 2008), fato semelhante ao observado neste estudo. Os funcionários analisados, que apresentavam maior intensidade da dor na escala visual analógica, também observou-se maiores diferenças de temperatura nas regiões dolorosas. Nos exemplos 1, 2, 5 e 7, o $\Delta T$ variou entre 0,5 (sugestivo de anormalidade) e 1,7 (anormalidade significativa) e intensidade de dor na EVA entre 6 (nível moderado) e 10 (nível intenso). Portanto, as medições de temperatura podem ser úteis como complementação na avaliação e documentação da disfunção autonômica em pacientes com quadro álgico.

No trabalho de Magas et al. (2014), verificaram a viabilidade da aplicação da termografia no diagnóstico de tendinite de punho comparando dois grupos: um grupo composto por indivíduos com presença de lesão e um grupo composto por indivíduos sem a presença de lesão e sem dor (grupo controle), comparada com o exame clínico. Para isso, avaliaram as diferenças térmicas dos pontos dolorosos do punho com tendinite e compararam com o membro contralateral (sadio). Para o grupo controle foi avaliada se haviam diferenças térmicas entre a mão dominante e o membro contralateral. Posteriormente, as alterações termográficas foram comparadas com o exame clínico. Os principais achados nas alterações das taxas de variações $(\Delta T)$ e na diferença média de temperatura, além dos valores da sensibilidade e especificidade, apresentaram resultados significativos indicando 
que a termografia foi capaz de detectar os pontos dolorosos e a diferença de temperatura para o lado não lesionado, identificando, assim, a alteração funcional que pode sugerir o diagnóstico de tendinite de punho.

É importante ressaltar, que alterações funcionais podem ser identificadas sem relato de queixas de dor, sendo um indicativo de possível lesão futura. Este método tem sido usado com sucesso na prevenção de lesões ortopédicas principalmente na área esportiva. (BALCI et al., 2016; BARBOSA, 2014). A Termografia representa a possibilidade de identificar a dor, a partir de uma resposta fisiológica, evitando a subjetividade do relato de queixa de dor dos funcionários na hora do exame.

\section{CONCLUSÃO}

A imagem termográfica é uma boa indicadora de dor comparada à avaliação subjetiva de queixa de dor dos funcionários em tratamento na fisioterapia ortopédica e traumatológica. $A$ resposta do sistema nervoso na presença de dor pode auxiliar no diagnóstico diferencial quanto a sua identificação, evitando a subjetividade do indivíduo. A termografia pode ser uma ferramenta utilizada pelo terapeuta no acompanhamento da evolução do quadro de dor durante o tratamento fisioterápico. Mais estudos são necessários no segmento de prevenção de futuras lesões, mapeamento dos pacientes e acompanhamento da intervenção escolhida.

\section{REFERÊNCIAS BIBLIOGRÁFICAS}

ANGELOTTI, G., SARDÁ J.R., J. J. Avaliação Psicológica da Dor. Dor \& Saúde Mental, p. 51-65, 2005.

BALCI G. A., BASARAN, T., COLAKOGLU, M. Analysing visual pattern of skin temperature during submaximal and maximal exercises. Infrared Physics \& Technology, v. 74, p. 57-62, 2016.

BARBOSA, F. A. P. Anthropometric thermal assessment method for early injuries in athletes. 2014.

BRIOSCHI, M. L., MALAFAIA, O. VARGAS, J. V. C. Review of recent developments in thermographic applications in health care. Inframation Proceedings, Estados Unidos, v. 5, pp. 918, 2004.

BRIOSCHI, M. L., COLMAN, D. Estudo da dor por imagem infravermelha. Rev Dor, v. 6, n. 3, p. 589-99, 2005.

BRIOSCHI, M. L., YENG, L. T., PASTOR, E. M. H., TEIXEIRA, M. J. Utilização da imagem infravermelha em reumatologia. Rev. Bras. Reumatol., São Paulo, v. 47, n. 1, p. 42-51, Feb. 2007.

BRIOSCHI, M. L., ABRAMAVICUS, S., CORREAA, C. F. Valor da imagem infravermelha na avaliação da dor. Rev Dor, v. 6, n. 1, p. 514-24, 2005.

BRIOSCHI, M. L., YENG, L. T., TEIXEIRA, M. J. Diagnóstico avançado em dor por imagem infravermelha e outras aplicações. Prática Hospitalar, v. 50, n. 1, p. 93-8, 2007.

CHAPMAN, C. R., CASEY, K. L., DUBNER, R., FOLEY, K. M., GRACELY, R. H. \& READING, A. E. Pain measurement: an overview. Pain, v. 22, n. 1, p. 1-31, 1985. 
DE MEIRA, L. F., KRUEGER, E., NEVES, E. B., NOHAMA, P., \& DE SOUZA, M. A. Termografia na área biomédica. Pan American Journal of Medical Thermology, v. 1, n. 1, p. 31-41, 2014.

DEYO R.A., MIRZA S.K., MARTIN B.I. Back pain prevalence and visit rates: estimates from U.S. national surveys, 2002. Spine (Phila Pa 1976) 2006;

FERREIRA G.D., SILVA M.C., ROMBALDI A.J., WREGE E.D., SIQUEIRA F.V., HALLAL P.C. Prevalência de dor nas costas e fatores associados em adultos do sul do Brasil: estudo de base populacional. Rev Bras Fisioter 2011; 15(1):31-36.

GEORGIOU, E., HADJIBALASSI, M., LAMBRINOU, E., ANDREOU, P. \& PAPATHANASSOGLOU, E.D. The Impact of Pain Assessment on Critically III Patients' Outcomes: A Systematic Review. BioMed research international. BioMed research international, v. 2015, 2015.

GOOdMAN, P. H., MURPHY, M. G., SILTANEN, G. L., KELLEY, M. P. \& RUCKER, L. Normal temperature asymmetry of the back and extremities by computer-assisted infrared imaging. Thermology, v. 1, n. 4, p. 194-202, 1986.

HOUDAS, Y., RING, E. F. J. Human body temperature: its measurement and regulation. Springer Science \& Business Media, 2013.

IASP, International Association for the Study of Pain. Disponível em: http://www.iasppain.org/Education/Content.aspx? ItemNumber=1698\&navltemNumber=576\}. Acesso em $10 \mathrm{de}$ abr. 2016.

LIMA MAG, ANDRADE AGM, BULCÃO CMA, MAGALHÃES FB, CARVALHO RCP, FREITAS SMC, SÁ SRFC, PORTO LA, NEVES RF. Programa de reabilitação de trabalhadores com LER/DORT do Cesat/Bahia: ativador de mudanças na Saúde do Trabalhador. Rev. bras. Saúde Ocup. São Paulo, 35 (121): 112-121, 2010.

LOVE, T. J. Thermography as an indicator of blood perfusion. Annals of the New York Academy of Sciences, v. 335 , n. 1, p. 429-437, 1980. Disponível em: http://onlinelibrary.wiley.com/doi/10.1111/j.1749-6632.1980.tb50766.x/abstract. Acesso em 10 de abr. 2016.

MARQUEZ, J. O. A dor e os seus aspectos multidimensionais. Ciência e Cultura, v. 63, n. 2, p. 2832, 2011.

MCCAFFERY, M., BEEBE, A. Pain in the elderly: special considerations. Pain: Clinical Manual for Nursing Practice, Mosby, USA, p. 308-323, 1989.

MILLS, S., TORRANCE, N., SMITH, B. H. Identification and Management of Chronic Pain in Primary Care: A Review. Current Psychiatry Reports, v. 18, n. 2, p. 1-9, 2016.

NEVES E.B., VILAÇA A.J., ROSA C., \& REIS V.M. Thermography in Neurologic Practice. The open neurology journal, v. 9, p. 24, 2015.

RILEY L.H., RICHTER C.P. Uses of the electrical skin resistance method in the study of patients with neck and upper extremity pain. The John Hopkins Med J 1975; 137(2):69-74

SCOPEL, E., ALENCAR, M., CRUZ, R. M. Medidas de avaliação da dor. Lecturas: Educación física y deportes, n. 105, p. 34, 2007.

TAKAHASHI MABC, SIMONELLI AP, SOUSA HP, MENDES RWB, ALVARENGA MVA. Programa de reabilitação profissional para trabalhadores com incapacidades por LER/DORT: relato de experiência do Cerest-Piracicaba, SP. Rev. bras. Saúde ocup., São Paulo, 35 (121): 100-111, 2010.

TEIXEIRA, M. J., YENG, L. T., KAZIYAMA, H. H. S. \& RAMOS, C. A. Fisiopatologia da dor músculo-esquelética. Revista de Medicina, v. 80, n. spe1, p. 63-77, 2001. 